\title{
Structural basis for channelling mechanism of a fatty acid $\beta$-oxidation multienzyme complex
}

\section{Momoyo Ishikawa, Daisuke Tsuchiya, Takuji Oyama, Yasuo Tsunaka and Kosuke Morikawa*}

Biomolecular Engineering Research Institute, Furuedai, Suita, Osaka, Japan

The atomic view of the active site coupling termed channelling is a major subject in molecular biology. We have determined two distinct crystal structures of the bacterial multienzyme complex that catalyzes the last three sequential reactions in the fatty acid $\beta$-oxidation cycle. The $\alpha_{2} \beta_{2}$ heterotetrameric structure shows the uneven ring architecture, where all the catalytic centers of 2-enoyl-CoA hydratase (ECH), L-3-hydroxyacyl-CoA dehydrogenase (HACD) and 3-ketoacyl-CoA thiolase (KACT) face a large inner solvent region. The substrate, anchored through the $3^{\prime}$-phosphate ADP moiety, allows the fatty acid tail to pivot from the ECH to HACD active sites, and finally to the KACT active site. Coupling with striking domain rearrangements, the incorporation of the tail into the KACT cavity and the relocation of $3^{\prime}$-phosphate ADP bring the reactive C2-C3 bond to the correct position for cleavage. The $\alpha$ helical linker specific for the multienzyme contributes to the pivoting center formation and the substrate transfer through its deformation. This channelling mechanism could be applied to other $\beta$-oxidation multienzymes, as revealed from the homology model of the human mitochondrial trifunctional enzyme complex.

The EMBO Journal (2004) 23, 2745-2754. doi:10.1038/

sj.emboj.7600298; Published online 1 July 2004

Subject Categories: structural biology; cellular metabolism Keywords: atomic structure; channelling mechanism; domain rearrangement; fatty acid $\beta$-oxidation; multienzyme complex

\section{Introduction}

Recent biochemical studies suggest that many enzymes are organized into multifunctional enzyme complexes in the cytoplasm or subcellular organelles. In these biomolecular assemblies, sequential catalytic reactions proceed through the transfer of the intermediates between individual component enzymes, without diffusion into the bulk aqueous medium. This reaction mode, termed the channelling mechanism, appears to be employed in various cellular processes. In fact, some gentle techniques for cell disruption show that many enzymes are assumed to form complex

${ }^{*}$ Corresponding author. Department of Structural Biology, Biomolecular Engineering Research Institute (BERI), 6-2-3 Furuedai, Suita, Osaka 565-0874, Japan. Tel.: + 8166872 8211; Fax: + 8166872 8210;

E-mail: morikawa@beri.or.jp

Received: 28 January 2004; accepted: 4 June 2004; published online: 1 July 2004 organizations, such as those involved in glycolysis and the citrate and urea cycles, highlighting the critical roles of the channelling in their catalytic reactions (Agius and Sherratt, 1997). Despite its importance and universality in cellular mechanisms, a structural basis to account for this efficient enzymic mechanism has not been established yet, although the molecular system for the pyruvate dehydrogenase multienzyme complex with covalently bound intermediates has been investigated in considerable detail (Milne et al, 2002). In particular, enzymes that participate in fatty acid metabolism, such as fatty acid synthesis and $\beta$-oxidation, tend to be assembled to confer kinetic advantages. Of all others, the molecular machinery for fatty acid $\beta$-oxidation is the subject of intense investigation, because this is an important catabolic process by which most organisms use fatty acids as energy and carbon sources. The sequential $\beta$-oxidation reactions shorten the saturated acyl-CoA by two carbons in each step to finally produce acetyl-CoA (Ac-CoA) (Figure 1A). The most obvious case for channelling is found in bacteria, such as Escherichia coli and Pseudomonas fragi (Pf) (Kunau et al, 1995). The trifunctional enzyme (TFE) from mammalian mitochondria was also suggested to involve channelling, because of the absence of detectable $\beta$-oxidation intermediates (Kunau et al, 1995; Yao and Schulz, 1996; Liang et al, 2001), although inconsistent cases were also reported (Eaton et al, 1999). Defects of mitochondrial fatty acid $\beta$-oxidation lead to several well-known metabolic disorders, such as Reye syndrome, cardiomyopathy and sudden infant death syndrome (SIDS) (Roe and Ding, 2001). The maintenance of high levels of mitochondrial $\beta$-oxidation could reduce the excessive fat accumulation and storage leading to human obesity, and thus an understanding of the molecular mechanism of $\beta$-oxidation is important from a pharmacological viewpoint.

The fatty acid $\beta$-oxidation spiral involves four enzymic activities, acyl-CoA dehydrogenase (ACD), 2-enoyl-CoA hydratase (ECH), L-3-hydroxyacyl-CoA dehydrogenase (HACD) and 3-ketoacyl-CoA thiolase (KACT) (Kim and Battaile, 2002) (Figure 1A). The bacterial and mitochondrial fatty acid $\beta$-oxidation multienzyme complexes predominantly exhibit the last three of these four enzymic activities. This bacterial fatty acid $\beta$-oxidation multienzyme (FOM) complex is composed of two $\alpha$ - and two $\beta$-subunits, which form a heterotetrameric $\alpha_{2} \beta_{2}$ structure. Genetic and biochemical analyses revealed that the ECH and HACD activities reside within the $\alpha$-subunit, whereas the KACT activity is harbored by the $\beta$ subunit (Binstock et al, 1977; DiRusso, 1990; Nakahigashi and Inokuchi, 1990; He Yang et al, 1991; Sato et al, 1992). In particular, the PfFOM complex, designated as HDT, was biochemically well characterized (Imamura et al, 1990). We successfully crystallized this complex purified from an E. coli overexpression system (Ishikawa et al, 1997).

Here, we report two atomic structures, which provide detailed insights into the substrate recognition and the catalytic mechanism. The three-dimensional structural view of 

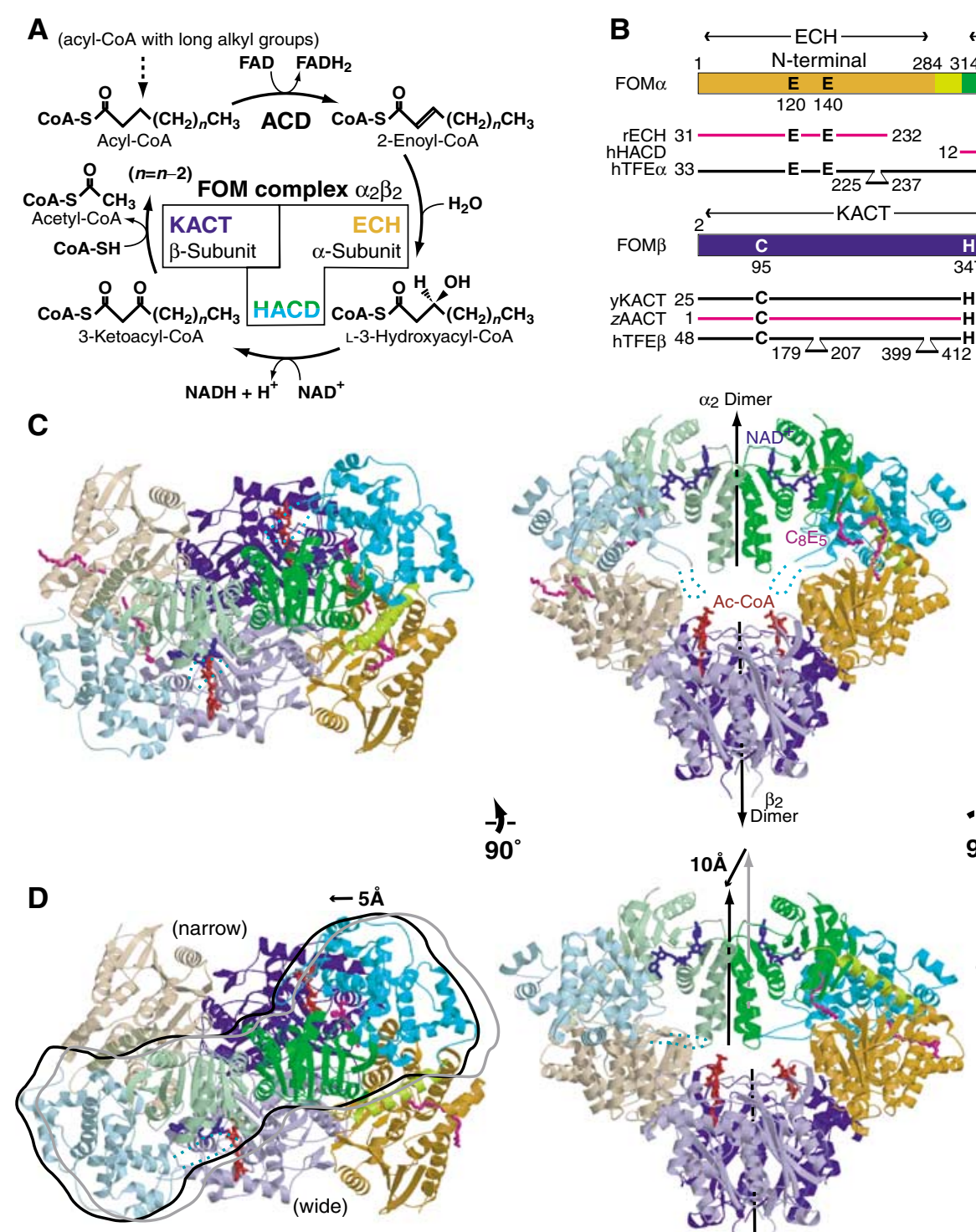

$90^{\circ}$
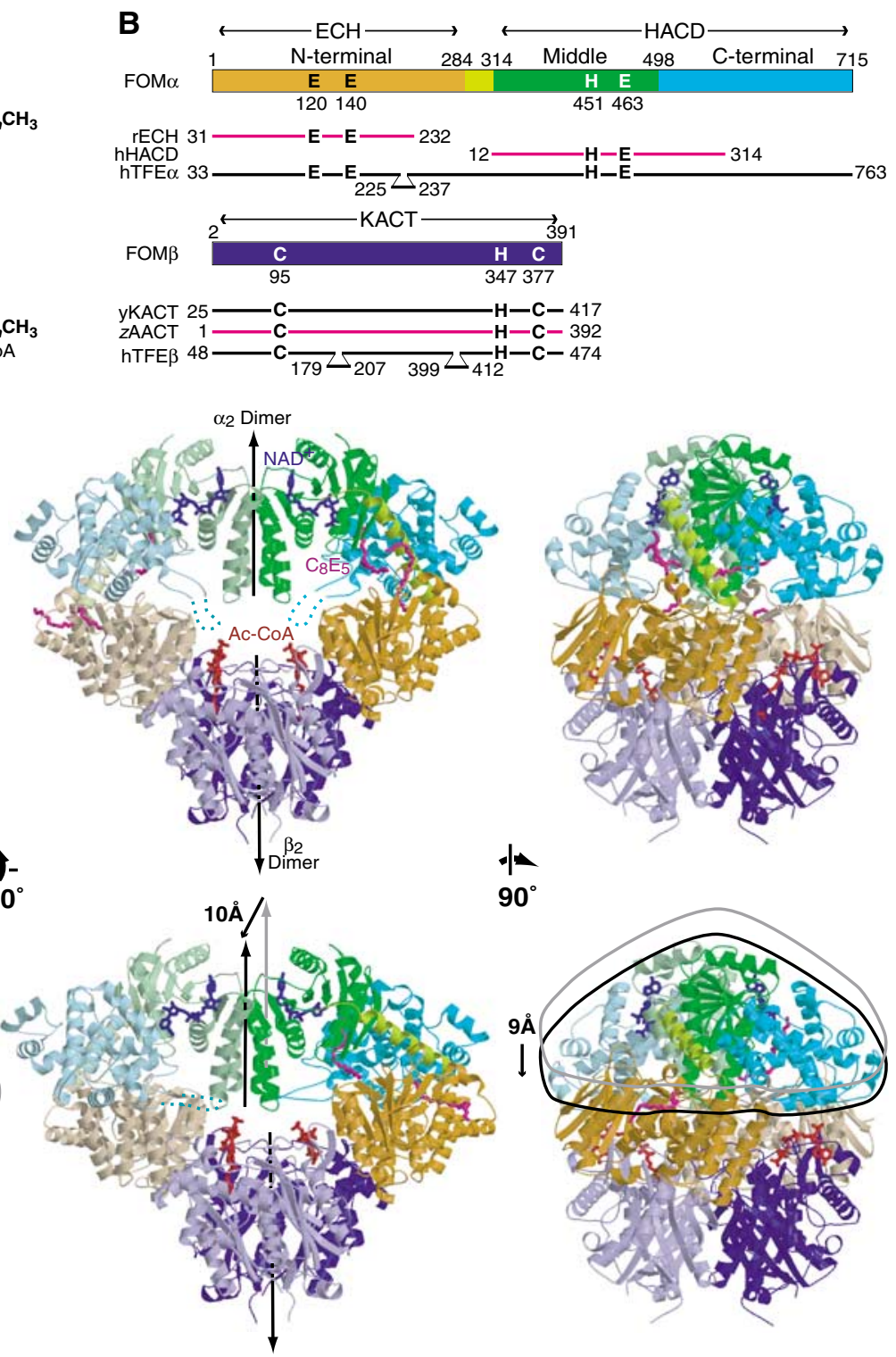

$90^{\circ}$
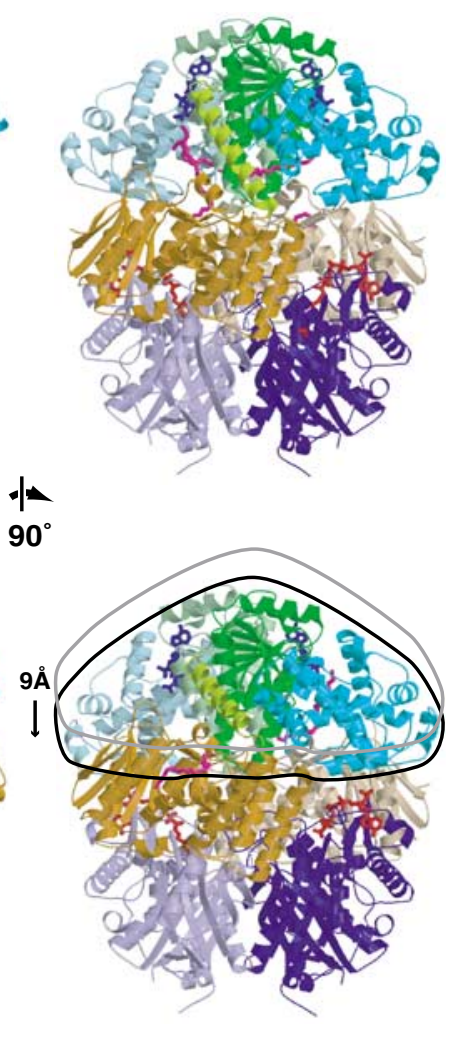

Figure $1 \beta$-Oxidation spiral and structure of FOM complex. (A) Catalytic scheme of $\beta$-oxidation spiral. (B) The subunit and domain organization of FOM compared with those of the hTFE complex, and the four monofunctional enzymes, rECH, hHACD, yKACT and $z$ AACT. In the $\alpha$-subunit, the $\mathrm{N}$-terminal domain is shown in brown, the $\alpha$-helical linker is yellow-green, the middle is green and the C-terminus is cyan, with the $\beta$-subunit colored purple. The same color code is applied for all subsequent diagrams. Conserved catalytic residues are labelled using the single-letter code. (C) Symmetric Form I structure. Each subunit is distinguished by dark ( $\alpha 1$ and $\beta 1)$ and light coloring ( $\alpha 2$ and $\beta 2)$. Three ligands, $\mathrm{NAD}^{+}$(dark blue), $\mathrm{C}_{8} \mathrm{E}_{5}$ (magenta) and Ac-CoA (red), are bound to each active site of the enzyme components. (D) Asymmetric Form II structure. The conversion of Form I to II is accompanied by large translations, as indicated by the bold arrows with lengths. It also generates the large difference ( 24 and $37 \AA$ ) in cleft sizes (top view).

the entire multienzyme allows us to describe the mechanistic details of the channelling mode for $\beta$-oxidation. The homology model building based on this complex structure also revealed notable structural features of the human mitochondrial TFE, which is directly linked to serious inherited metabolic disorders.

\section{Results and discussion}

\section{Architecture of the multienzyme complex}

The crystal of the PfFOM complex exhibits remarkable polymorphism, which appears to be ascribed to its unsteady domain assembly. The addition of a fatty acid tail analog, n-octylpentaoxyethylene $\left(\mathrm{C}_{8} \mathrm{E}_{5}\right)$, was essential to improve the diffraction quality and stability of the crystals, implying that the binding of this ligand may have reinforced the fragile architecture that is inevitable for multienzymes. We determined two crystal structures, designated as Form I and II (Table I). The final models of both structures comprise all of the residues of the intact complex molecule, except for an unstructured segment of the $\alpha$-chain and also contain three ligand molecules bound to different sites. Both crystal forms contain one intact FOM complex molecule with the heterotetrameric $\alpha_{2} \beta_{2}$ subunit structure in the asymmetric unit. The Form I crystal structure possesses a noncrystallographic twofold axis shared by both $\alpha_{2}$ and $\beta_{2}$ homodimers, in agreement 
Table I Data collection, phasing and refinement

\begin{tabular}{|c|c|c|c|c|c|c|c|}
\hline & \multicolumn{3}{|c|}{ Form I } & \multirow{2}{*}{$\begin{array}{l}\text { Form II } \\
\text { Native4 }\end{array}$} & \multicolumn{3}{|c|}{ Form I } \\
\hline & Native1 & Native2 & Native3 & & $\mathrm{CH}_{3} \mathrm{HgCl}$ & $\mathrm{C}_{2} \mathrm{H}_{5} \mathrm{HgCl}$ & SeMet \\
\hline \multicolumn{8}{|l|}{ Data collection } \\
\hline Wavelength $(\AA)$ & 1.2500 & 1.0000 & 1.0000 & 1.5418 & 1.0000 & 1.5418 & 0.9794 \\
\hline Resolution $(\AA)$ & 2.8 & 2.5 & 3.8 & 3.5 & 3.8 & 4.0 & 2.8 \\
\hline \multicolumn{8}{|l|}{$R_{\text {merge }}$} \\
\hline Total & 0.068 & 0.086 & 0.124 & 0.152 & 0.071 & 0.091 & 0.076 \\
\hline Highest & 0.215 & 0.271 & 0.236 & 0.316 & 0.123 & 0.133 & 0.204 \\
\hline \multicolumn{8}{|l|}{$I / \sigma(I)$} \\
\hline Total & 21.0 & 7.2 & 5.9 & 8.2 & 7.6 & 9.3 & 19.3 \\
\hline Highest & 7.3 & 2.7 & 3.2 & 4.5 & 2.9 & 6.3 & 8.5 \\
\hline \multicolumn{8}{|l|}{ Completeness } \\
\hline Total & 98.5 & 95.5 & 98.0 & 99.8 & 97.4 & 80.1 & 100 \\
\hline Highest & 97.4 & 80.1 & 98.5 & 99.8 & 96.8 & 77.6 & 100 \\
\hline \multicolumn{8}{|l|}{ Phasing } \\
\hline Phasing power (centric/acentric) & & & & & $1.0 / 1.3$ & $0.94 / 1.2$ & $0.84 / 0.88$ \\
\hline Figure of merit & 0.31 & & & & & & \\
\hline \multicolumn{8}{|l|}{ Refinement } \\
\hline & \multicolumn{2}{|c|}{ Form I } & Form II & & & & \\
\hline & Native2 & Native3 & Native4 & & & & \\
\hline Resolution $(\AA)$ & $20-2.5$ & $20-3.8$ & $12-3.5$ & & & & \\
\hline \multicolumn{8}{|l|}{$R_{\mathrm{work}}$} \\
\hline Total & 0.202 & 0.214 & 0.233 & & & & \\
\hline Highest & 0.291 & 0.229 & 0.257 & & & & \\
\hline \multicolumn{8}{|l|}{$R_{\text {free }}{ }^{\mathrm{a}}$} \\
\hline Total & 0.244 & 0.262 & 0.282 & & & & \\
\hline Highest & 0.386 & 0.290 & 0.291 & & & & \\
\hline R.m.s.d. bond length $(\AA)$ & 0.007 & 0.005 & 0.003 & & & & \\
\hline R.m.s.d. bond angle (deg) & 1.2 & 0.98 & 1.0 & & & & \\
\hline
\end{tabular}

${ }^{\mathrm{a}} R_{\text {free }}$ was calculated for $7 \%$ of reflections randomly excluded from the refinement.

with the previous electron microscopic analysis (Ishikawa et al, 1997) (Figure 1C). However, Form II exhibits a strikingly deformed architecture, where the two-fold axis of the $\alpha_{2}$ homodimer moves $5 \AA$ away from the $\beta_{2}$ homodimer. Simultaneously, the upper half of the $\alpha_{2}$ dimer approaches the $\beta_{2}$ dimer by $9 \AA$ along the two-fold axis. As a result, $10 \AA$ rigid-body movement of the upper half takes place between Form I and II. In spite of this large movement, all of the functional domains retain essentially the same conformation (Figure 1D).

The individual component enzymes of the FOM complex share $30-40 \%$ amino-acid identities with the corresponding monofunctional enzymes from various sources. In addition to the structural similarity between the two types of each enzyme component, the positions of three ligands, Ac-CoA, nicotinamide adenine dinucleotide $\left(\mathrm{NAD}^{+}\right.$) and a substrate mimic, $\mathrm{C}_{8} \mathrm{E}_{5}$, allow us to describe clear three-dimensional structural views of the fatty acid $\beta$-oxidation complex, including the domain organization and the catalytic sites of the individual component enzymes.

In Form I, the intact complex structure reveals the uneven ring architecture, where the saddle-shaped $\alpha_{2}$ dimeric subunits (designated as $\alpha 1$ - and $\alpha 2$-chains) are spirally mounted on the tightly connected $\beta_{2}$ dimeric subunits ( $\beta 1$ - and $\beta 2$ - chains) lying at the bottom of the ring (Figure 1C). The $\alpha$-subunit, which bears the ECH and HACD activities, is structurally divided into the $\mathrm{NH}_{2}(\mathrm{~N})$-terminal, middle and $\mathrm{COOH}$ (C)-terminal domains (Figure 1B). A long $\alpha$-helix (residues 284-309) in the $\alpha$-subunit connects the $\mathrm{N}$-terminal ECH domain with the HACD region, which is composed of the middle $\mathrm{NAD}^{+}$-binding and $\mathrm{C}$-terminal domains. The $\beta$ subunit with the KACT activity comprises two compact core domains and a loop domain, as found in the crystal structures of the monofunctional degradative thiolase from Saccharomyces cerevisiae (yKACT) (Mathieu et al, 1994, 1997 ) and the biosynthetic thiolase from Zoogloea ramigera (zAACT) (Modis and Wierenga, 1999, 2000). The three component enzymes in the complex retain essentially the same architectures as those in the crystal structures of the monofunctional enzymes (Engel et al, 1996; Modis and Wierenga, 1999; Barycki et al, 2000).

One major interface in the complex resides in the middle $\mathrm{NAD}^{+}$-binding domain of HACD, which occupies most of the saddle. A helix-turn-helix unit of the middle domain forms a four-helix bundle structure, through a mixture of polar and hydrophobic interactions, to stabilize the $\alpha_{2}$ dimer. The twofold axis runs through the four-helix bundle across the large solvent region at the complex center and toward the extensive 
interface of the $\beta_{2}$ dimer. Consequently, the two ECH domains occupy the farthest positions from the two-fold axis and lack direct contact with each other. The contact between the KACT $\beta$-subunits is contributed by plenty of polar and hydrophobic interactions, including an inter-subunit $\beta$-sheet, as found in the crystal structures of the degradative (Mathieu et al, 1994) and biosynthetic thiolases (Modis and Wierenga, 1999). In contrast to these two interfaces contributed by secondary structures, the contacts between the $\alpha$ - and $\beta$-subunits, unique to this multienzyme complex, appear to be loose and to play a versatile role in the maintenance of the complex. The $\alpha$-helical end and the loop of the ECH domain in the $\alpha$-subunits separately interact with the distinct protruded loops of the individual KACT subunits, and thus the dimeric KACT subunits jointly anchor a single $\alpha$-subunit. The buried surface areas were calculated to be $3200 \AA^{2}$ for the $\alpha_{2}$ dimer, $3800 \AA^{2}$ for the $\beta_{2}$ dimer and $4200 \AA^{2}$ in total for the $\alpha-\beta$ contact, respectively, which are all statistically defined as 'large interfaces' (Conte et al, 1999).

The internal architectures of all of the domains are essentially identical between Form I and II. On the other hand, the arrangements of the middle and C-terminal domains, constituting HACD, remarkably differ between the two forms (Figures 1C and D), whereas the domain arrangements of the KACT $\beta$-subunit are unchanged. Intriguingly, these domain rearrangements appear to be coupled with the conformational change of the multienzyme-specific $\alpha$-helical linker between ECH and HACD. Notably, this long $\alpha$-helix is partly disrupted upon the conversion from Form I to II. Consequently, in Form II, the tip of the four-helical bundle of HACD shifts toward the $\beta_{2}$ dimer by a $10 \AA$ translation (Figure 1D). This $\alpha$-helical deformation appears to be connected with the dissociation of two $\mathrm{C}_{8} \mathrm{E}_{5}$ molecules: in Form I (Figure 1C), the multienzyme contains four $\mathrm{C}_{8} \mathrm{E}_{5}$ molecules, two of which are equivalently bound to each HACD substratebinding site of the $\alpha$-subunits related by two-fold symmetry. By contrast, in the ECH domain, two $\mathrm{C}_{8} \mathrm{E}_{5}$ molecules occupy the inner and outer halves of the long hydrophobic tunnel, respectively (Figure 2A). On the other hand, in Form II (Figure 1D), neither the ECH nor HACD active sites in one $\alpha$-subunit bind $\mathrm{C}_{8} \mathrm{E}_{5}$ molecules, whereas their active sites in the other $\alpha$-subunit are fully occupied by the ligands. This implies a strong link between the deformed structure of Form II and the asymmetric ligand binding. Taken together, the linker $\alpha$-helix in the multienzyme appears to play a key role in the positional relationships between the catalytic centers, through its conformational change. Thus, the dynamic domain behavior through the versatile linker could account for the coordinated catalytic mechanism of $\beta$-oxidation. This notion is compatible with the finding that the addition of $\mathrm{C}_{8} \mathrm{E}_{5}$ substantially reduces the combined specific activity of $\mathrm{ECH}$ and HACD in the $\alpha$-subunit, presumably because it prevents the domain rearrangement.

It should also be noted that the complex structure in Form I generates the large and irregular solvent regions with rough dimensions of $65 \AA \times 35 \AA \times 30 \AA$. All of the catalytic sites of the three enzyme components face this inner solvent region and occupy positions nearest to each other. This overall feature is reminiscent of the morphology proposed for the mammalian fatty acid synthases, aside from the differences in the molecular symmetry and the arrangements of enzymic components (Smith, 1994; Brink et al, 2002).
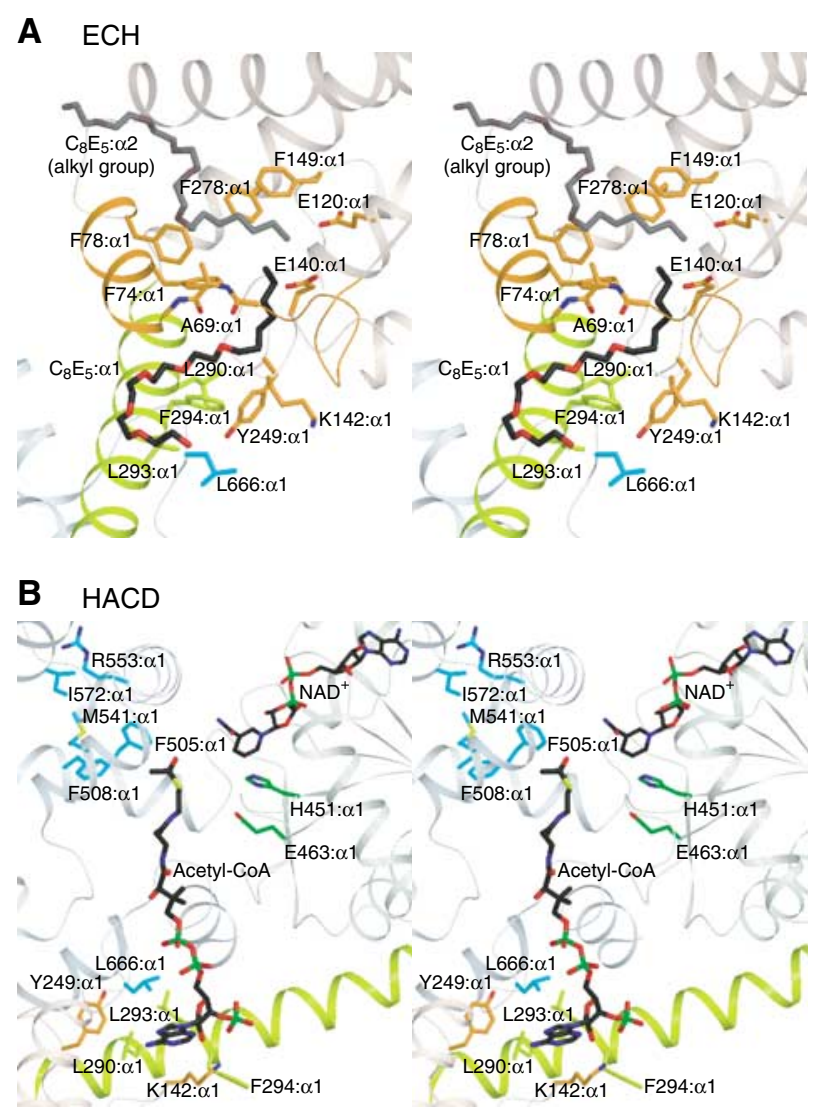

C KACT

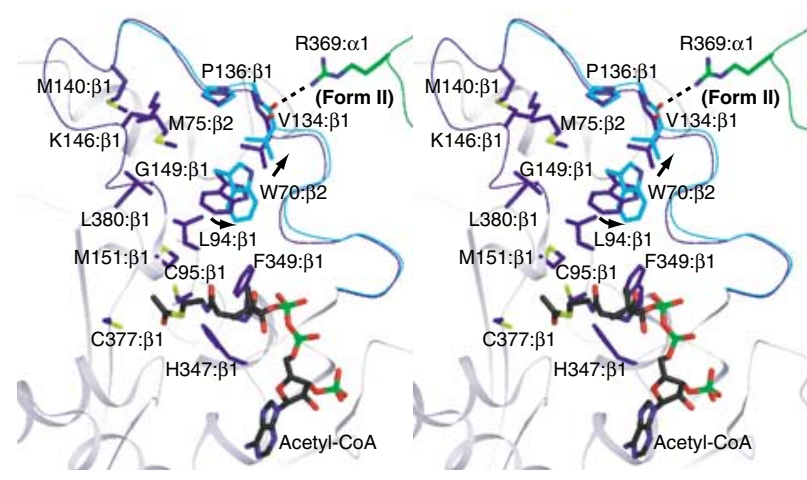

Figure 2 Active sites of three FOM components. Stereo diagrams show catalytic and hydrophobic residues around ligands in Form I. (A) Two $\mathrm{C}_{8} \mathrm{E}_{5}$ molecules bound to each $\mathrm{ECH}$ of the dimer $(\alpha 1$ and a2) in different modes, 'inside' (clear gray) and 'outside' (faint gray). The alkyl groups of two $\mathrm{C}_{8} \mathrm{E}_{5}$ molecules are trapped with hydrophobic residues. Some parts of main chains (light brown) represent identical portions to rECH (Engel et al, 1996). (B) Ac-CoA and $\mathrm{NAD}^{+}$molecules bound to HACD in the Native3 crystal. The acetyl group of Ac-CoA points into the hydrophobic tunnel. (C) Ac-CoA molecules bound to the two KACT subunits. In Form II, the interaction of Arg369 with Val134 causes the $1 \AA$ A elevation (arrow) of the loop containing Val134 and Pro136 (cyan), and the subsequent rotation of the Trp70: $\beta 2$ side chain (cyan).

\section{2-Enoyl-CoA hydratase}

The N-terminal ECH domain (residues 1-283) in the $\alpha$-subunit (Figure 1B) is essentially identical to the hexameric ECH from rat liver mitochondria $(\mathrm{rECH})$ with a root mean square deviation (r.m.s.d.) value of $1.7 \AA$ (Figure $3 \mathrm{~A}$ ), except for the 
A

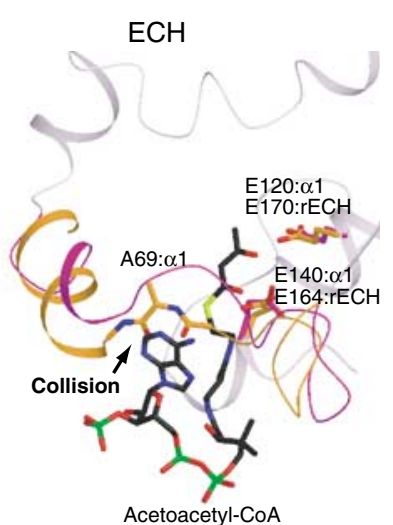

D

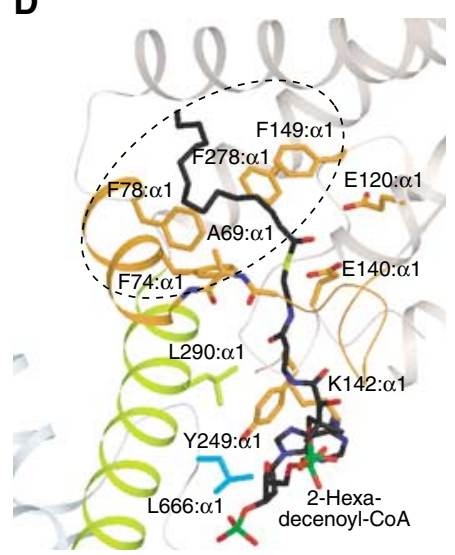

B

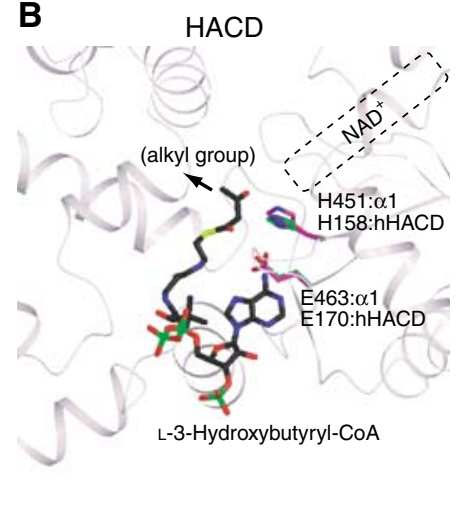

C

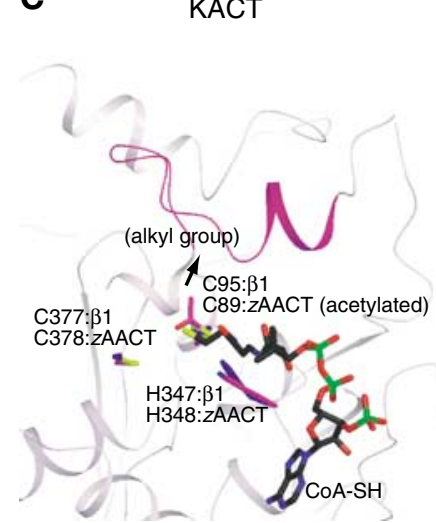

E

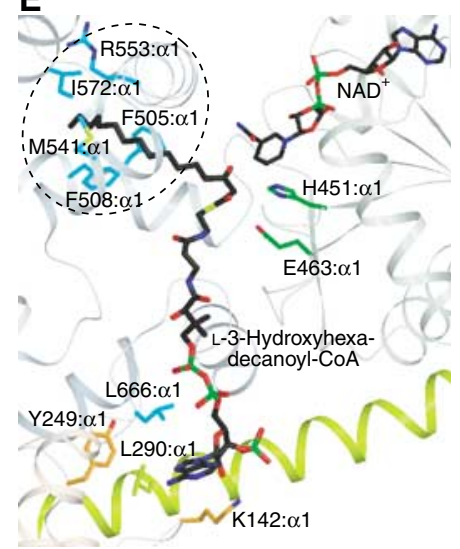

$\mathbf{F}$

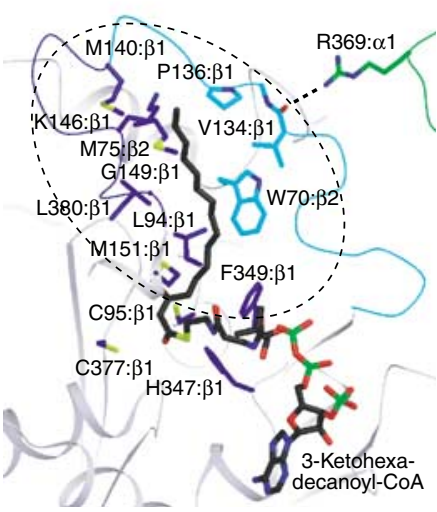

Figure 3 Active sites and substrate binding. (A-C) Superimposition of FOM components onto the corresponding monofunctional enzymes, highlighting its different structural portions (magenta). (A) Collision between the adenine base of AcAc-CoA bound to rECH (Engel et al, 1996) and Ala69 of ECH (arrow). (B) Two catalytic residues and L-3-hydroxybutyryl-CoA bound to hHACD (Barycki et al, 2000). The NAD ${ }^{+}$-binding site is vacant in the hHACD structure (dotted line). The arrow denotes the extension of an alkyl chain. (C) Three catalytic residues and the CoA bound to monofunctional zAACT (Modis and Wierenga, 1999), which are identical to those in KACT. (D-F) Putative binding mode of substrates. (D) 2-Hexadecenoyl-CoA model docked onto the FOM structure. Its long alkyl group is incorporated into the hydrophobic tunnel (dashed oval), while its $3^{\prime}$-phosphate ADP is bound to the pocket composed of a part of HACD and the $\alpha$-helical linker. (E) L-3Hydroxyhexadecanoyl-CoA model denoted that ECH and HACD share the adenine base-binding pocket (D). The long alkyl group of the substrate is surrounded by hydrophobic residues (dashed oval). (F) 3-Ketohexadecanoyl-CoA model docked onto the binding site, in concert with the conformational change of Trp70 as in Figure 2C. The long alkyl group of the substrate is encompassed by the hydrophobic residues (dashed oval).

C-terminal region (residues 208-283) corresponding to the T2 subdomain of rECH (Engel et al, 1996). Although both of the C-terminal T2 subdomains from the monofunctional $\mathrm{rECH}$ and the ECH component fold into the all- $\alpha$ structure, their arrangements relative to the common major core, comprising the spiral fold and the T1 domain (Engel et al, 1996), are remarkably different from each other. The $\mathrm{ECH}$ component also possesses the additional five-turn $\alpha$-helix, the C-terminus of which is connected directly to the multienzyme-specific $\alpha$-helical linker.

In the hydrophobic catalytic pocket, the carboxyl side chains of two glutamates, Glu120 and Glu140, and the $\mathrm{H}_{2} \mathrm{O}$ molecule that participate in hydration of substrates adopt the same configuration as those observed in the crystal structures of $\mathrm{rECH}$ complexed with acetoacetyl-CoA (AcAc-CoA) (Figure 3A) (Engel et al, 1996), octanoyl-CoA (Engel et al, 1998) and 4-(N,N-dimethylamino) cinnamoyl-CoA (Bahnson et al, 2002), suggesting that the catalytic mechanism is identical between the monofunctional and multifunctional enzymes. In Form I, the substrate analogs, $\mathrm{C}_{8} \mathrm{E}_{5}$, were bound to different sites within each ECH domain of the two $\alpha$ subunits. In one $\alpha$-subunit, a $\mathrm{C}_{8} \mathrm{E}_{5}$ molecule is accommodated within the inner half of the elongated tunnel from the 'inside' of the central solvent region (Figure 2A). The recognition mode of this ligand appears to mimic the substrate binding in the monofunctional $\mathrm{rECH}$, as revealed from the good agreement in the two structures superimposed between the ligand molecule and the pantetheine part of the AcAc-CoA molecule (Figures 2A and 3A). By contrast, the same ligand, bound to the other subunit, appears to approach the ECH active site from the 'outside' of the complex (Figure 2A). The combination of these two structural features for ligand binding reveals the entire tunnel for a longer fatty acid tail (C16) of about $20 \AA$ long, which can be fully incorporated in the $\mathrm{ECH}$ component. The tail is trapped by van der Waals interactions with hydrophobic residues, such as Ala69, Phe74, Phe78, Phe149 and Phe278, which constitute the wall of the tunnel extended from the active site (Figures 2A and 3D). The polar moiety of $\mathrm{C}_{8} \mathrm{E}_{5}$ lies outside of $\mathrm{ECH}$, and weakly interacts with some side chains on the linker $\alpha$-helix (Figure 2A). On the 
other hand, we could not reasonably allocate the binding pocket for the $3^{\prime}$-phosphate ADP moiety by analogy with the monofunctional rECH complex with the ligands, AcAc-CoA (Engel et al, 1996) and octanoyl-CoA (Engel et al, 1998), because of the steric clash of its adenine base with the $\mathrm{N}$ terminus of an $\alpha$-helix of the ECH domain (Figure 3A).

\section{L-3-Hydroxyacyl-CoA dehydrogenase}

The HACD component (residues 314-715) is divided into the $\mathrm{NAD}^{+}$-binding domain (residues 314-497) and the C-terminal domain (residues 498-715) (Figure 1B). The $\mathrm{NAD}^{+}$ domain structure in the multienzyme is almost identical to that of the monofunctional human HACD (hHACD) with a $1.2 \AA$ r.m.s.d. value (Barycki et al, 2000) (Figure 3B). Despite the absence of a repeated sequence, the C-terminal polypeptide of HACD folds into the all- $\alpha$ architecture, where the two halves are related by the internal pseudo-two-fold symmetry. By contrast, the corresponding region in the monofunctional human (Barycki et al, 1999b, 2000, 2001) and pig HACD (Barycki et al, 1999a) consists of the two individual Cterminal segments, each of which constitutes half of the Cterminal domain structure in the HACD component. These two small all- $\alpha$ lobes contribute to forming the pig and human HACD homodimers with an exact two-fold symmetry. Interestingly, in the HACD component, the extra 10-residue segment, inserted between the two halves, constitutes the disordered loop (Figures 1C and D, dotted line), which extends toward the catalytic site of KACT. This difference in the C-terminal domain organization generates the apparently distinct architectures between the two types of HACD, whereas their internal structures are identical with a $1.7 \AA$ r.m.s.d. value (Figure 3B). In particular, the two prominent helix-turn-helix structures, lying most distantly in the monofunctional HACD dimer, form the tight dimer interface connecting the two $\alpha$-subunits in Pf HACD.

$\mathrm{NAD}^{+}$is bound to the typical $\alpha / \beta$ dinucleotide fold of the middle domain in the same mode as that in the monofunctional HACD, whereas the substrate is recognized in a different manner (Figures $2 \mathrm{~B}$ and $3 \mathrm{~B}$ ). In the Native3 crystal (Table I), the substrate analog, Ac-CoA, is bound to the $\alpha$ subunit at a sufficient occupancy to exhibit its environments (Supplementary data 1). The active site structure is well conserved between PfFOM and the monofunctional hHACD (Barycki et al, 1999b, 2000, 2001): His451 and Glu463 in FOM take the same configuration as those in hHACD. Likewise, $\mathrm{NAD}^{+}$, and acetyl and $\beta$-mercaptoethylamine moieties of AcCoA essentially lie at the same positions between the two enzymes. On the other hand, the $3^{\prime}$-phosphate ADP moiety in PfFOM occupies a different position from that in hHACD. The adenine base is accommodated into a hydrophobic pocket formed by Lys142 and Tyr249 in ECH, Leu290 in the $\alpha$-helical linker and Leu666 in HACD. The hydrophobic character of the pocket is conserved among the multifunctional enzymes (Supplementary data 2). Interestingly, the adenine-binding pocket lies at a position for substrates to contact not only HACD (Figure 3E) but also ECH (Figure 3D). Thus, we assume that this nucleotide-binding pocket could play the role as a pivoting center in transferring the pantetheine and acyl moieties from one active site to another during $\beta$-oxidation. Meanwhile, the $3^{\prime}$-phosphate moiety is located near some polar residues of the $\alpha$-helical linker and HACD. In addition, the methyl group of Ac-CoA accesses the hydro- phobic tunnel, consisting of Phe505, Phe508, Met541, Arg553 and Ile572 (Figure 2B). Longer fatty acyl chains appear more favorable for accommodation into this hydrophobic tunnel (Figure 3E). It should be noted that in Form $\mathrm{I}$ the $\mathrm{C}_{8} \mathrm{E}_{5}$ molecule can partly occupy the same position as Ac-CoA in the Native3 crystal, and its occupancy appears to vary from crystal to crystal.

The structural feature of Ac-CoA binding is consistent with the results from mutational analyses using spectrophotometric assay (Ishikawa et al, 1997): The double mutant, L290D/L293D, where both Leu290 and Leu293 were replaced by Asp, reduced the combined specific activity of ECH and HACD to about $14 \%$ that of the wild-type activity. On the other hand, two mutants, F294A and K142A, did not show significant reduction of the activity. These results suggest that the introduction of negative charges into the hydrophobic pocket seriously affects the substrate binding. Furthermore, the substrate binding can be retained, as far as the hydrophobic property of the pocket is not impaired.

\section{3-Ketoacyl-CoA thiolase}

The entire architecture of KACT, including the two core domains and a loop domain, is essentially identical to those of the monofunctional degradative (Mathieu et al, 1994, 1997) and biosynthetic thiolases (Modis and Wierenga, 1999, 2000), with $1.2 \AA$ r.m.s.d. values (Figure 3C). The conformational conservation of KACT, spanning the three domains, is remarkable in comparison with those in the $\mathrm{ECH}$ and the HACD components. Instead of AcAc-CoA added for co-crystallization, only Ac-CoA was observed in its active site (Figures $1 \mathrm{C}, \mathrm{D}$ and $2 \mathrm{C}$ ), suggesting that AcAcCoA was converted to Ac-CoA through a thiolase reaction during co-crystallization. On the other hand, the cleaved acetyl group, which is expected to be covalently bound to Cys95, as found in the biosynthetic thiolase (Modis and Wierenga, 1999), appears to be hydrolytically decomposed. The conformational view of the KACT active center, including the CoA moiety and the three catalytic residues, Cys95, His347 and Cys377, is identical to that of the monofunctional enzyme (Figure 3C) (Modis and Wierenga, 1999, 2000).

Previous biochemical data demonstrate that the thiolase reaction for long fatty acyl chains requires the $\alpha$-subunits (Sato et al, 1992; Ishikawa et al, 1997). In fact, the conversion from Form I to II allows Arg369, at the tip of the four-helical bundle structure of HACD, to approach and form the intersubunit hydrogen bond between Arg369 side-chain and the main-chain carbonyl oxygen of Val134 of one $\beta$-subunit. As a result, the segment (residues 130-137), lying near the loop responsible for the substrate specificity in the monofunctional thiolases (Mathieu et al, 1994, 1997; Modis and Wierenga, 1999, 2000), is raised by about $1.0 \AA$, and thereby causes a torsional rotation of the spatially adjacent Trp70 side chain in the counterpart of the KACT dimer (Figures 2C and 4B). The Trp70 side chain occupies the position that partitions the substrate-binding site into two portions for the CoA moiety and the acyl group (Figure $3 \mathrm{~F}$ ), and is located just behind the thiolytically cleaved $\mathrm{C} 2-\mathrm{C} 3$ bond of AcAc-CoA, as modelled by Mathieu et al (1997). The aromatic side chain appears to serve as a door in guiding the long fatty acyl chains of substrates into the elongated hydrophobic cavity, with walls composed of Met75: $\beta 2$, Leu94, Pro136, Met140, Lys146, Gly149, Met151, Phe349 and Leu380. The acyl chain 
A
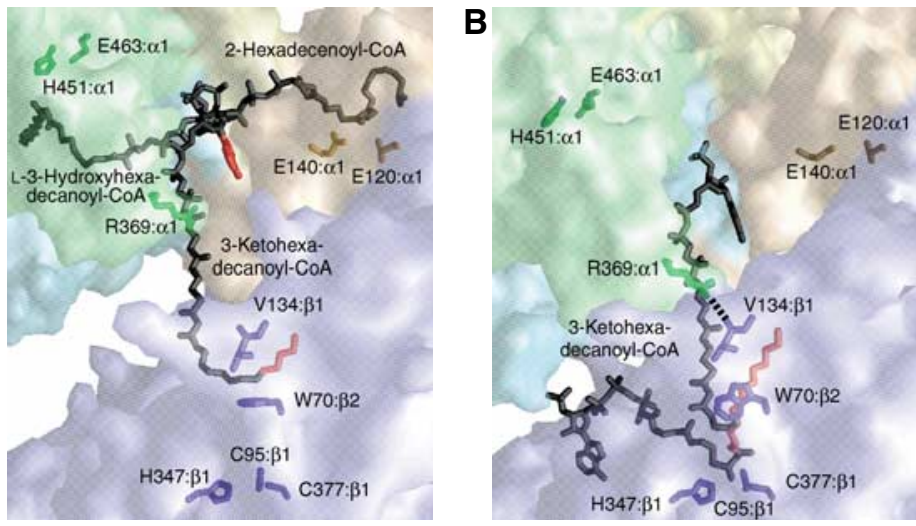

C
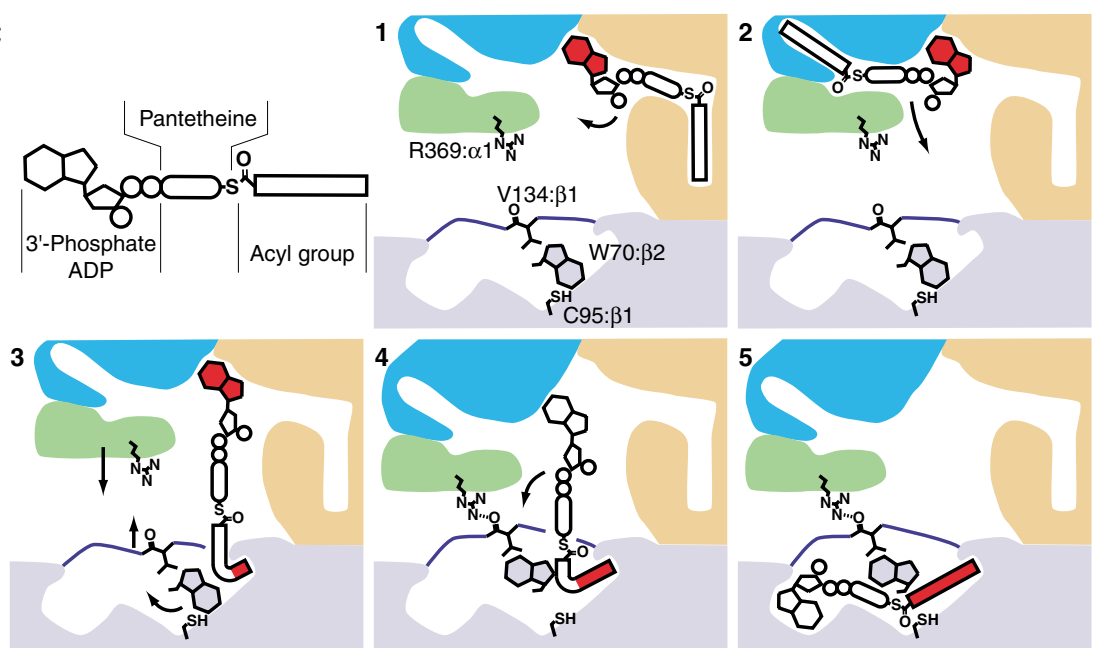

Figure 4 Channelling mode. (A) Transfer of the substrate from ECH to KACT through HACD in Form I. Key residues in each subunit are indicated to illustrate their roles. This representation corresponds to scenes 1-3 in (C). (B) The 3'-phosphate ADP of the KACT substrate transferred from the $\alpha$ - to the $\beta$-subunit in Form II. This view corresponds to scenes 4 and 5 in (C). (C) Schematic drawing of the channelling model. The adenine base and acyl group, each of which acts as a pivoting center during substrate transfer, are colored red. The arrows indicate directions of movements.

incorporation would induce rearrangements of the CoA moiety, resulting in the correct positioning of the target bond for the catalytic residues (Figures $3 \mathrm{~F}$ and $4 \mathrm{C}$ ).

\section{Channelling mechanism}

The substantial separation of the ECH, HACD and KACT catalytic centers urged us to consider the large mobility of the substrates or products within the multienzymes during coordinated catalytic reactions. In particular, the order of the three sequential reaction steps in $\beta$-oxidation (Figure $1 \mathrm{~A}$ ), apparently, does not fit to the multienzyme architecture, where ECH intervenes between the HACD and KACT enzymes. The multienzyme structure also reveals that the binding pocket of the $3^{\prime}$-phosphate ADP moiety is shared by ECH and HACD, whereas the binding site of the $3^{\prime}$ phosphate ADP moiety in KACT is independent of that of the former two enzymes (Figures 4A and B, and Supplementary data 3 ). In addition to these multienzymespecific positions of the CoA and acyl chain moieties, the rearrangements of the individual enzyme components, coupled with the conversion of Form I into II, allow us to propose a reasonable channelling scheme (Figure 4C), where 2-hexadecenoyl-CoA (C16) (2-enoyl-CoA) is a representative substrate for $\mathrm{ECH}$. The sharing of the single binding pocket for the adenine base by ECH and HACD implies the substrateanchored diffusion mechanism, where L-3-hydroxyhexadecanoyl-CoA (L-3-hydroxyacyl-CoA), generated through $\mathrm{ECH}$ hydration, is transferred to the HACD active site to produce 3-ketohexadecanoyl-CoA (3-ketoacyl-CoA). It is likely that the long acyl groups can travel between the ECH and HACD catalytic centers through their dynamic behavior, while fixing the adenine moiety to the common binding pocket, and thus the $\mathrm{C} 2$ and $\mathrm{C} 3$ reactive carbons of an intermediate can transfer to the HACD catalytic centers, without diffusing into the solvent (Figure 4A).

Next, the C16 fatty acid tail pivots toward KACT, but at most only its end can reach the hydrophobic cavity with Trp70 (Figures 4A and C3). Therefore, the complete transfer of the HACD product to KACT requires a drastic conformational transition, as revealed by the Form I and II structural differences, so that the reactive $\mathrm{C} 2-\mathrm{C} 3$ bond of the substrate can be correctly positioned at the KACT active site. The conformational transition simultaneously weakens the binding of the adenine base and thereby detaches it from the binding pocket (Figures 4B and C4). This notion is consistent with the finding that the adenine-binding pocket is deformed through rearrangements of HACD, ECH and the $\alpha$-helical linker in Form II. In particular, the striking deformation of 
the linker may be coupled with the substrate transfer. Subsequently, the $3^{\prime}$-phosphate ADP moiety is transferred from the binding pocket in the $\alpha$-subunit to the CoA-binding site in the KACT $\beta$-subunit, while keeping the long acyl chain moiety in the cavity (Figure 4C4-5). According to our model based on Form II (Figure 4B), it appears that the incorporation of sufficiently long acyl chains into the cavity is required to ensure the substrate transfer. However, it is possible that closer approach of the $\alpha$-subunit to the $\beta$-subunit allows acyl chains to be firmly anchored during actual reaction. Then, KACT catalyzes the cleavage reaction of 3-ketohexadecanoylCoA to generate tetradecanoyl-CoA (acyl-CoA) and Ac-CoA. This channelling mode contrasts with that of fatty acid synthase (Smith, 1994), the substrate of which is covalently bound to the acyl carrier protein. In the $\beta$-oxidation spiral, a failure in anchoring the acyl chains might lead to the leakage of fatty acid substrates, depending on their lengths. This notion is in good agreement with the 'leaky-hosepipe' model proposed for mitochondrial fatty acid $\beta$-oxidation (Stanley and Tubbs, 1975; Liang et al, 2001). The complete $\beta$-oxidation spiral requires the involvement of $\mathrm{ACD}$, and thus it is intriguing to consider its direct interaction with FOM, given the high protein concentration within cells.

\section{Human trifunctional enzyme model}

The sequence similarity of human mitochondrial TFE to the PfFOM complex (33\% identity and $53 \%$ similarity for $\alpha$ subunit, 38 and $59 \%$ for $\beta$-subunit) allowed us to construct its homology model (Figure 5), which contains residues 33763 for the $\alpha$-subunit and $48-474$ for the $\beta$-subunit, aside from the three inserted segments (residues 226-236 in $\alpha$ subunit, $171-211$ and $399-413$ in $\beta$-subunit) (Figure 1B). The model is based on the $\alpha_{2} \beta_{2}$ subunit structure (Carpenter et al, 1992) rather than $\alpha_{4} \beta_{4}$ (Uchida et al, 1992), because the latter may be modelled by dimerizing the former molecule. Each of the three dimer interfaces $(\alpha-\alpha, \beta-\beta$ and $\alpha-\beta)$ in the model represents reasonable amino-acid interactions. In particular, the structural features of the interface between the $\alpha$ - and $\beta$ subunits, with Phe217 surrounded by several hydrophobic residues (Figure 5A, inset), nicely explain how the mutation of Val282 to Asp in the $\alpha$-subunit causes the TFE deficiency (Ibdah et al, 1998).

The TFE model exhibits a biased distribution of positive charges (Figure 5B), whereas PfFOM shows a relatively uniform distribution. Notably, the basic residues, assembled around the central solvent region, are conserved among various TFE complexes. An unmodelled insertion (residues 171-211) with 10 basic residues coincidentally protrudes into the central solvent region (Figure 5A), although its functional significance remains unclear. The cluster of basic residues may contribute to localizing TFE on the inner mitochondrial membrane, through electrostatic interactions with the negative charges on the lipid bilayer. The positive charges may also function to electrostatically attract ACD by interacting with the putative negative patches.

\section{Conclusions}

The distinct atomic structures of the $\beta$-oxidation multienzyme complex revealed by this crystallographic study appear to reflect two scenes among various architectures. Depending on this conformational versatility inherent in
A
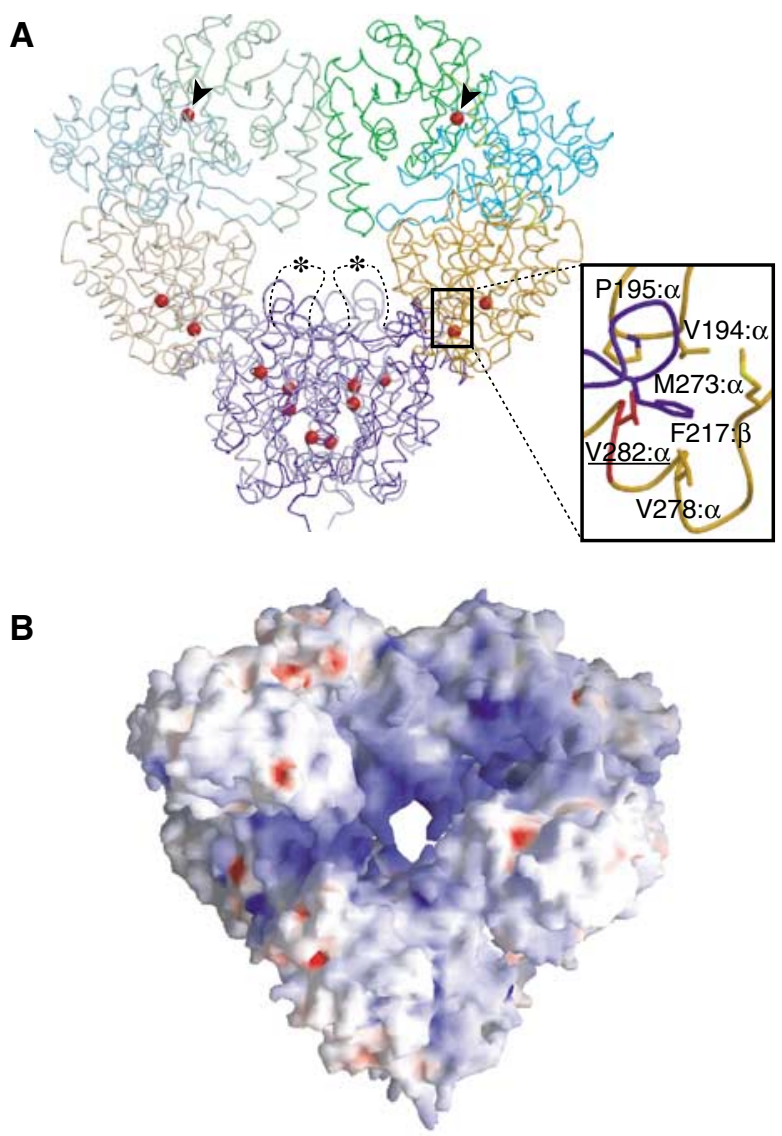

Figure 5 Homology model of the human TFE complex. (A) The symmetric TFE architecture, with the mutation sites (red spheres) relevant to various genetic diseases (Ibdah et al, 1998; Eaton et al, 2000). The arrowheads denote the HACD active sites. The inset indicates Val282, located in the interface between ECH in the $\alpha$-subunit and KACT in the $\beta$-subunit. The insertion specific for TFE is depicted by dotted lines with asterisks. (B) Electrostatic surface representation showing the biased distribution of positive charges around the central solvent region.

bacterial and mitochondrial $\beta$-oxidation multienzymes, the functional domains are competently rearranged to accomplish the smooth transfer of substrates and products between different active sites. A similar architectural transformation coupled with substrate transfer could be employed by multienzymes in other metabolic systems, such as the tricarboxylic acid cycle, the urea cycle and glycolytic pathway, which proceed via efficient and sequential catalytic reactions, without complete equilibrium with the intermediate pool in the surrounding media.

\section{Materials and methods}

Crystallization, structure determination and refinement Wild-type and mutant S40C: $\beta$ (Ser40 to Cys on the $\beta$-subunit) were overproduced and purified, according to a slightly modified version of the previous procedure (Ishikawa et al, 1997). The selenomethionine-substituted protein was prepared by the addition of seleno-DL-methionine to methionine assay medium (DIFCO). Before crystallization, the FOM protein was dialyzed against a solution containing $10 \mathrm{mM}$ PIPES (pH 6.5), $0.6 \%$ (w/v) $\mathrm{C}_{8} \mathrm{E}_{5}$ and $3 \mathrm{mM}$ DTT. The crystallization solution contained $5-10 \mathrm{mg} / \mathrm{ml}$ protein, $82 \mathrm{mM}$ MES (pH 5.5), $1 \mathrm{mM} \mathrm{NAD}{ }^{+}, 0.1 \mathrm{mM} \mathrm{AcAc-CoA}$ and $3.5 \%$ PEG 4000. Drops, equilibrated against a reservoir with higher salt and 
PEG 4000 at $20^{\circ} \mathrm{C}$ by vapor diffusion, yielded Form II crystals: space group $\mathrm{P} 22_{1} 2_{1}$ with unit cell dimensions, $a=95.7 \AA, b=118.4 \AA$, $c=191.4 \AA$. The addition of $1 \mathrm{mM} \mathrm{ZnCl}_{2}$ to the same crystallization solution provided Form I: space group $\mathrm{C} 2$ with unit cell dimensions, $a=180.8 \AA, b=94.7 \AA, c=160.6 \AA, \beta=111.6^{\circ}$. The $\mathrm{Zn}$ atoms (not shown in Figure 1C) were located between the two FOM molecules, stabilizing the crystal packing in this form. Mercury derivatives were prepared by co-crystallization with the addition of $0.5 \mu \mathrm{l}$ of a saturated $\mathrm{CH}_{3} \mathrm{HgCl}$ or $\mathrm{C}_{2} \mathrm{H}_{5} \mathrm{HgCl}$ solution to $8.5 \mu \mathrm{l}$ of crystallization solution, containing the mutant or wild-type protein, respectively. Crystals were flash-frozen in cryoprotectant, containing $82 \mathrm{mM}$ MES (pH 5.5), $1 \mathrm{mM} \mathrm{NAD}{ }^{+}, 0.1 \mathrm{mM}$ AcAc-CoA, $12.5 \%$ PEG 4000, $10 \%$ glycerol and optionally $1 \mathrm{mM} \mathrm{ZnCl}$. The Native3 crystal, containing Ac-CoA bound to the $\alpha$-subunit, was grown under the identical crystallization condition as that for Form I, and found to possess essentially the same crystallographic data. However, the occupancy of this ligand bound to the $\alpha$-subunit varied between the Native1-3 crystals. In the Native 1 and 2 crystals, $\mathrm{C}_{8} \mathrm{E}_{5} \mathrm{~s}$ were observed in place of Ac-CoA. Besides, two other crystal forms were obtained: space group $\mathrm{P} 2{ }_{1} 2_{1} 2_{1}$ with unit cell dimensions, $a=96.1 \AA, b=153.6 \AA$, $c=198.0 \AA$, and space group $\mathrm{C} 2$ with unit cell dimensions, $a=179.1 \AA, \quad b=93.5 \AA, c=149.3 \AA, \quad \beta=111.9^{\circ}$. Their structures were solved at 3.9 and $3.3 \AA$, respectively. These structures were essentially identical to that of Form I, except for differences in crystal packing.

X-ray diffraction data were collected with $\mathrm{CuK} \alpha$ or synchrotron source at BL38B1 and BL40B2 in SPring-8 (Hyogo, Japan) at $100 \mathrm{~K}$ and were processed with HKL (Otwinowski and Minor, 1997) and MOSFLM (Leslie, 1991). The structure of Form I was solved by the multiple isomorphous replacement method using SHARP (de La Fortelle and Bricogne, 1997). The initial model was built on the electron density map using Native1 data, which were later swapped with Native2 during the structure refinement using CNS (Brünger et al, 1998). The density modification by noncrystallographic symmetry averaging and solvent flattening procedures significantly improved experimental electron density, and allowed us to complete the chain tracing. The structure of Form II was determined by the molecular replacement method. The analysis using AMoRe (Navaza, 1994) provided a solution when the molecular structure in Form I was separated into three domains (ECH, HACD and KACT) for the search probes. The structure refinement was carried out with CNS (Brünger et al, 1998). The final models of both structures comprise all of the residues of the intact complex molecule, except for a few missing loops (Form I, residues 594-600: $\alpha 1$ and 596599: 2 ; Form II, 593-601: $\alpha 2$; Figures $1 \mathrm{C}$ and D, dotted line), and also contain three ligand molecules bound to different sites. Figures

\section{References}

Agius L, Sherratt HSA (eds) (1997) Channelling in Intermediary Metabolism. London, UK: Portland Press Ltd

Bahnson BJ, Anderson VE, Petsko GA (2002) Structural mechanism of enoyl-CoA hydratase: three atoms from a single water are added in either an E1cb stepwise or concerted fashion. Biochemistry 41: 2621-2629

Barycki JJ, O’Brien LK, Birktoft JJ, Strauss AW, Banaszak LJ (1999a) Pig heart short chain L-3-hydroxyacyl-CoA dehydrogenase revisited: sequence analysis and crystal structure determination. Protein Sci 8: 2010-2018

Barycki JJ, O’Brien LK, Bratt JM, Zhang R, Sanishvili R, Strauss AW, Banaszak LJ (1999b) Biochemical characterization and crystal structure determination of human heart short chain L-3-hydroxyacyl-CoA dehydrogenase provide insights into catalytic mechanism. Biochemistry 38: 5786-5798

Barycki JJ, O’Brien LK, Strauss AW, Banaszak LJ (2000) Sequestration of the active site by interdomain shifting. $J$ Biol Chem 275: 27186-27196

Barycki JJ, O’Brien LK, Strauss AW, Banaszak LJ (2001) Glutamate 170 of human L-3-hydroxyacyl-CoA dehydrogenase is required for proper orientation of the catalytic histidine and structural integrity of the enzyme. $J$ Biol Chem 276: 36718-36726

Binstock JF, Pramanik A, Schulz H (1977) Isolation of a multienzyme complex of fatty acid oxidation from Escherichia coli. Proc Natl Acad Sci USA 74: 492-495 were generated with the programs MOLSCRIPT (Kraulis, 1991), Raster3D (Merrit and Bacon, 1997) and GRASP (Nicholls et al, 1991).

\section{Homology model building}

The homology model of hTFE was constructed on the basis of Form I structure of $P F F O M$, referring to the amino-acid sequence alignment in Supplementary data 2. The alignment was initially generated with program CLUSTAL W (Thompson et al, 1994), and further improved by visual inspection of the PfFOM structure superimposed onto each of rECH (PDB ID: 1dub), hHACD (PDB ID: 1f12), yKACT (PDB ID: 1afw) and $z$ AACT (PDB ID: 1qfl) structures The models of the $\alpha$ - and $\beta$-subunits, containing 720 and 371 residues respectively, were constructed with program Insight II (Accerlys Inc., USA). The unmodelled N-terminal regions in both subunits contain the signal sequences for mitochondrial transport, which would be presumably unstructured. The remaining unmodelled segments are located at the peripheral regions, except for residues $171-211$ of the $\beta$-subunit (Figure $5 A$ ). Prior to the model regularization, the $\mathrm{N}$ - and $\mathrm{C}$-terminal residues of the unmodelled segments were artificially protected with acetyl and methylamide groups, respectively, which mimic missing peptide bonds and charges at the termini. The structural model was energetically optimized with program DISCOVER (Accerlys Inc., USA) using the CVFF force field, until unreasonable steric collisions were minimized.

\section{Protein Data Bank accession numbers}

The coordinates for the Native2-4 crystals have been deposited in the Protein Data Bank with ID codes 1WDK, 1WDM and 1WDL, respectively.

\section{Supplementary data}

Supplementary data are available at The EMBO Journal Online.

\section{Acknowledgements}

We acknowledge Naoto Yagi, Keiko Miura and Nobutaka Shimizu for help in data collection at SPring-8. We thank Hiroyuki Toh, Narutoshi Kamiya and Hisako Ichihara for homology model building of the human TFE complex. We are grateful to Akihiko Kawaguchi and Shigeyuki Imamura for providing the pHDT4 plasmid and the substrates for the PfFOM complex. This work was supported in part by the New Energy and Industrial Technology Development Organization (NEDO) of the Japanese Government.

Brink J, Ludtke SJ, Yang C-Y, Gu Z-W, Wakil SJ, Chiu W (2002) Quaternary structure of human fatty acid synthase by electron cryomicroscopy. Proc Natl Acad Sci USA 99: 138-143

Brünger AT, Adams PD, Clore GM, DeLano WL, Gros P, GrosseKunstleve RW, Jiang JS, Kuszewski J, Nilges M, Pannu NS, Read RJ, Rice LM, Simonson T, Warren GL (1998) Crystallography \& NMR system: a new software suite for macromolecular structure determination. Acta Crystallogr D 54: 905-921

Carpenter K, Pollitt RJ, Middleton B (1992) Human liver long-chain 3-hydroxyacyl-coenzyme A dehydrogenase is a multifunctional membrane-bound beta-oxidation enzyme of mitochondria. Biochem Biophys Res Commun 183: 443-448

Conte LL, Chothia C, Janin J (1999) The atomic structure of protein-protein recognition sites. J Mol Biol 285: 2177-2198

de La Fortelle E, Bricogne G (1997) Maximum-likelihood heavyatom parameter refinement for multiple isomorphous replacement and multiwavelength anomalous diffraction methods. Methods Enzymol 276: 472-494

DiRusso CC (1990) Primary sequence of the Escherichia coli fad BA operon, encoding the fatty acid-oxidizing multienzyme complex, indicates a high degree of homology to eucaryotic enzymes. J Bacteriol 172: 6459-6468

Eaton S, Bartlett K, Pourfarzam M (1999) Intermediates of myocardial mitochondrial beta-oxidation: possible channelling of $\mathrm{NADH}$ and of CoA esters. Biochim Biophys Acta 1437: 402-408 
Eaton S, Bursby T, Middleton B, Pourfarzam M, Mills K, Johnson AW, Barlett K (2000) The mitochondrial trifunctional protein: centre of a $\beta$-oxidation metabolon? Biochem Soc Trans 28: $177-182$

Engel CK, Kiema TR, Hiltunen JK, Wierenga RK (1998) The crystal structure of enoyl-CoA hydratase complex with octanoyl-CoA reveals the structural adaptations required for binding of a long chain fatty acid-CoA molecule. J Mol Biol 275: 847-859

Engel CK, Mathieu MJ, Zeelen P, Hiltunen JK, Wierenga RK (1996) Crystal structure of enoyl-coenzyme A (CoA) hydratase at $2.5 \AA$ resolution: a spiral fold defines the CoA-binding pocket. EMBO J 15: $5135-5145$

He Yang X-Y, Schulz H, Elzinga M, Yang S-Y (1991) Nucleotide sequence of the promoter and $f a d B$ gene of the $f a d B A$ operon and primary structure of the multifunctional fatty acid oxidation protein from Escherichia coli. Biochemistry 30: 6788-6795

Ibdah JA, Tein I, Dionisi-Vici C, Bennett MJ, IJlst L, Gibson B, Wanders RJA, Strauss AW (1998) Mild trifunctional protein deficiency is associated with progressive neuropathy and myopathy and suggests a novel genotype-phenotype correlation. J Clin Invest 102: 1193-1199

Imamura S, Ueda S, Mizugaki M, Kawaguchi A (1990) Purification of the multienzyme complex for fatty acid oxidation from Pseudomonas fragi and reconstitution of the fatty acid oxidation system. J Biochem 107: 184-189

Ishikawa M, Mikami Y, Usukura J, Iwasaki $H$, Shinagawa $H$, Morikawa K (1997) Reconstitution, morphology and crystallization of a fatty acid $\beta$-oxidation multienzyme complex from Pseudomonas fragi. Biochem J 328: 815-820

Kim J-JP, Battaile KP (2002) Burning fat: the structural basis of fatty acid $\beta$-oxidation. Curr Opin Struct Biol 12: 721-728

Kraulis PE (1991) MOLSCRIPT: a program to produce both detailed and schematic plots of protein structures. J Appl Crystallogr 24: 946-950

Kunau W-H, Dommes V, Schulz H (1995) $\beta$-Oxidation of fatty acids in mitochondria, peroxisomes, and bacteria: a century of continued progress. Prog Lipid Res 34: 267-342

Leslie AGW (1991) Macromolecular data processing. In Crystal Computing $V$, Moras D, Pogjarny AD, Thierry JC (eds) pp 2738. Oxford, UK: Oxford University Press

Liang X, Le W, Zhang D, Schulz H (2001) Impact of the intramitochondrial enzyme organization on fatty acid oxidation. Biochem Soc Trans 29: 279-282

Mathieu M, Modis Y, Zeelen JPh, Engel CK, Abagyan RA, Ahlberg A, Rasmussen B, Lamzin VS, Kunau W-H, Wierenga RK (1997) The $1.8 \AA$ crystal structure of the dimeric peroxisomal 3-ketoacyl-CoA thiolase of Saccharomyces cerevisiae: implication for substrate binding and reaction mechanism. J Mol Biol 273: 714-728

Mathieu M, Zeelen JPh, Pauptit RA, Erdmann R, Kunau W-H, Wierenga RK (1994) The $2.8 \AA$ crystal structure of peroxisomal
3-ketoacyl-CoA thiolase of Saccharomyces cerevisiae: a fivelayered $\alpha \beta \alpha \beta \alpha$ structure constructed from two core domains of identical topology. Structure 2: 797-808

Merrit EA, Bacon DJ (1997) Raster3D version 2.0: a program for photorealistic molecular graphics. Methods Enzymol 277: 505-524

Milne JLS, Shi D, Rosenthal PB, Sunshine JS, Domingo GJ, Wu X, Brooks BR, Perham RN, Henderson R, Subramaniam S (2002) Molecular architecture and mechanism of an icosahedral pyruvate dehydrogenase complex: a multifunctional catalytic machine. EMBO J 21: 5587-5598

Modis Y, Wierenga RK (1999) A biosynthetic thiolase in complex with a reaction intermediate: the crystal structure provides new insights into the catalytic mechanism. Structure 7: 1279-1290

Modis Y, Wierenga RK (2000) Crystallographic analysis of the reaction pathway of Zoogloea ramigera biosynthetic thiolase. J Mol Biol 297: 1171-1182

Nakahigashi K, Inokuchi H (1990) Nucleotide sequence of the fadA and fadB genes from Escherichia coli. Nucleic Acids Res 18: 4937

Navaza J (1994) AMoRe: an automated package for molecular replacement. Acta Crystallogr A 50: 157-163

Nicholls A, Sharp KA, Honig B (1991) Protein folding and association: insights from the interfacial and thermodynamic properties of hydrocarbons. Proteins Struct Funct Genet 11: 281-296

Otwinowski Z, Minor W (1997) Processing of X-ray diffraction data collected in oscillation mode. Methods Enzymol 276: 307-325

Roe CR, Ding J (2001) Mitochondrial fatty acid oxidation disorders. In The Metabolic and Molecular Bases of Inherited Disease, Scriver CR, Beaudet AL, Sly WS, Valle D (eds) 8 edn, Vol. 2, pp 22972326. New York: McGraw-Hill

Sato S, Hayashi M, Imamura S, Ozeki Y, Kawaguchi A (1992) Primary structure of the genes, fao $A$ and $f a o B$, from Pseudomonas fragi B-0771 which encode the two subunits of the HDT multienzyme complex involved in fatty acid $\beta$-oxidation. $J$ Biochem 111: 8-15

Smith S (1994) The animal fatty acid synthase: one gene, one polypeptide, seven enzymes. FASEB J 8: 1248-1259

Stanley KK, Tubbs PK (1975) The role of intermediates in mitochondrial fatty acid oxidation. Biochem $J$ 150: 77-88

Thompson JD, Higgins DG, Gibson TJ (1994) CLUSTAL W: improving the sensitivity of progressive multiple sequence alignment through sequence weighting, position-specific gap penalties and weight matrix choice. Nucleic Acids Res 22: 4673-4680

Uchida Y, Izai K, Orii T, Hashimoto T (1992) Novel fatty acid $\beta$-oxidation enzymes in rat liver mitochondria. J Biol Chem 267: 1034-1041

Yao KW, Schulz H (1996) Intermediate channeling on the trifunctional beta-oxidation complex from pig-heart mitochondria. J Biol Chem 271: 17816-17820 